HEART REVIEW

\title{
Pregnancy in women with valvular heart disease
}

\author{
Karen K Stout, Catherine M Otto
}

Patients with valvular disease who desire pregnancy or are already pregnant require specialised care. Ideally, women undergo preconceptual counselling that addresses any procedures needed to decrease the risks of pregnancy, including valve replacement, if the patient has symptoms at baseline. Management during pregnancy includes replacing any contraindicated medications with safer alternatives, optimising loading conditions, careful monitoring and aggressive treatment of any exacerbating factors. Rarely, percutaneous or surgical intervention is required during pregnancy. Labour and delivery often require invasive haemodynamic monitoring and a multi-disciplinary team for optimal maternal and fetal outcomes.

See end of article for authors' affiliations

.......................

Correspondence to: Dr K K Stout, Division of Cardiology, Box 356422, University of Washington, 1959 Pacific NE, Rm AA522, Seattle, WA 98195, USA;

stoutk@u.washington.edu

Accepted 21 June 2006 Published Online First 11 August 2006

.......................

www.heartjnl.com
Heart 2007;93:552-558. doi: 10.1136/hrt.2005.067975

and concerns about valve deterioration in those with tissue prostheses. ${ }^{4}$

In women with valvular disease, the normal haemodynamic changes of pregnancy can precipitate cardiac symptoms in previously stable women, or may exacerbate symptoms in those who had symptoms before pregnancy. Ideally, preconceptual planning includes: (1) advice to each woman about the risk of pregnancy for herself and the baby, (2) optimisation of her cardiac condition, and (3) institution of careful monitoring and treatment starting before conception and continuing through pregnancy into the postpartum period. Unfortunately, some women are first diagnosed with valvular disease due to haemodynamic decompensation during pregnancy, and many with known valvular disease present for medical evaluation only after they are already pregnant. Evaluation and treatment of these patients can be challenging, although many can still have successful pregnancies. ${ }^{5}$

\section{HAEMODYNAMICS OF PREGNANCY}

Pregnancy causes significant haemodynamic changes that progress through pregnancy and further change during labour and delivery and in the postpartum period. ${ }^{6}$ During pregnancy, cardiac demands increase due to the placental circulation and hormonal effects, with a $30-50 \%$ increase in cardiac output, a 10-20 bpm increase in heart rate and a $30-50 \%$ increase in blood volume. Systemic vascular resistance decreases so that blood pressure remains low, despite the increase in cardiac output. Owing to the increase in blood volume with an unchanged red cell mass, there is a fall in haematocrit. ${ }^{7-9}$ Most of these haemodynamic changes begin early in the first trimester, peak during the second trimester and reach a plateau phase in the third trimester (fig 1).

During labour and delivery, there is an increase in cardiac output, heart rate, blood pressure and systemic vascular resistance, all of which are accentuated with each contraction. ${ }^{710}{ }^{11}$ Pain and anxiety contribute to the increase in heart rate and blood pressures, such that pain control and anxiolytics help reduce the acuity of the haemodynamic changes. Delivery of the placenta increases afterload by removing the low-resistance vascular bed, and rapidly increases preload with venous return of blood to the maternal circulation. Blood loss may result in further decrease in the haematocrit. These changes pose a substantial demand on cardiac function in a patient with valvular disease, sometimes necessitating invasive haemodynamic monitoring and aggressive medical

Abbreviation: UFH, unfractionated 
Box 1 Valvular disease in women of childbearing age

Valve stenosis

- Aortic stenosis

- Congenital

- Recurrent after valvotomy in childhood

- Subaortic membrane

- Mitral stenosis

- Rheumatic

- Congenital

- Pulmonic stenosis

- Congenital

- Isolated pulmonic valve stenosis

- Tetralogy of Fallot

Valve regurgitation

- Aortic regurgitation

- Congenital (including bicuspid valve)

- Rheumatic

- Connective tissue disorder (eg, Marfan's syndrome)

- Mitral regurgitation

- Myxomatous

- Congenital

- Rheumatic

- Pulmonic regurgitation

- Residual after surgical intervention for tetralogy of Fallot or pulmonary stenosis

treatment in the peripartum period. In a delivery complicated by excessive blood loss, infection, arrhythmia or complex obstetric issues, these demands are further amplified.

\section{DIAGNOSTIC EVALUATION}

Initial assessment of a woman with valvular disease who is contemplating pregnancy or is already pregnant focuses on the history and physical examination. Ideally, the history includes information on any prior diagnostic testing including echocardiograms and exercise stress tests, as well as any relevant records of percutaneous or surgical interventions. Baseline exercise tolerance is an important predictor of the ability to tolerate pregnancy, regardless of the underlying lesion. Many of the normal symptoms of pregnancy are also symptoms of cardiac decompensation. However, while dyspnoea on exertion, orthopnoea, ankle oedema and palpitations are expected, symptoms of angina, resting dyspnoea, paroxysmal nocturnal dyspnoea or a sustained arrhythmia are not normal, even in pregnancy (box 2).

The normal flow murmur of pregnancy is typically soft (grade 1 or 2), located at the pulmonic region, associated with a normal first and second heart sound, and is not accompanied by a diastolic murmur or signs of heart failure. Echocardiography is indicated in women with a history of valvular or congenital heart disease, significant dyspnoea, any signs of heart failure, a grade 3 or greater systolic murmur, or any diastolic murmur. Echocardiography provides information regarding the aetiology and severity of valvular disease, as well as ventricular function and pulmonary artery pressures.

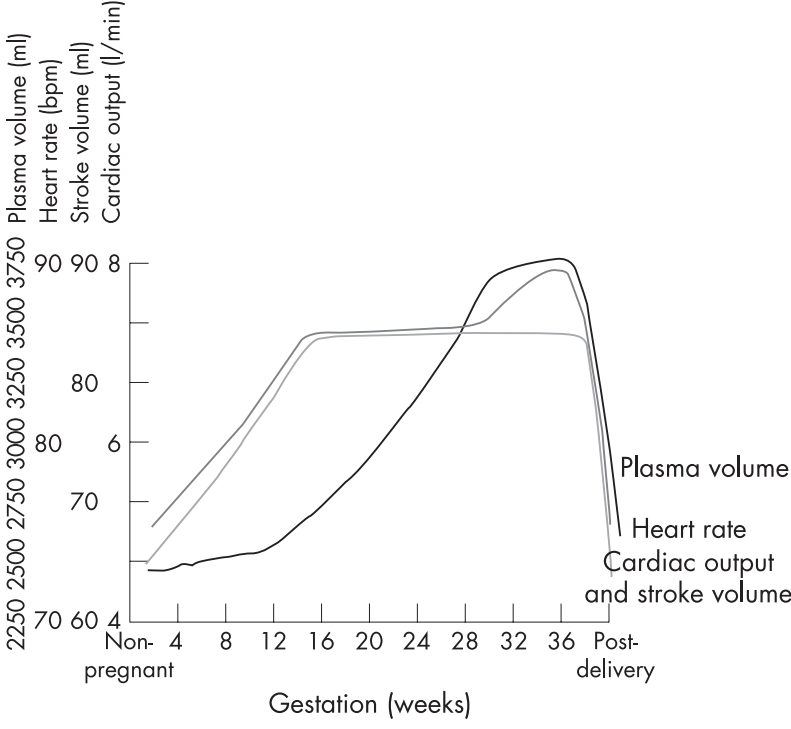

Figure 1 Physiological changes in pregnancy. Fall in systemic and pulmonary vascular resistance during pregnancy. Blood pressure may fall in the second trimester, rising slightly in later pregnancy. Note that cardiac output and stroke volume peak by 16 weeks of gestation. (Adapted from Thorne. ${ }^{6}$ )

\section{GENERAL MANAGEMENT OF PREGNANT WOMEN WITH VALVULAR DISEASE}

Women with valvular disease may not tolerate the haemodynamic changes of pregnancy or labour and delivery, even when asymptomatic before pregnancy (box 3). In addition, some patients with only mild valve dysfunction before pregnancy develop acute valvular dysfunction-for example, acute mitral regurgitation owing to chordal rupture of acute stenosis of a thrombosed mechanical prosthesis.

The first step in management of women with valvular disease is to establish the level of maternal and fetal risk, on the basis of functional status, severity and type of valvular disease, left ventricular function and pulmonary pressures (box 4).

In addition to the medical issues of pregnancy, the inheritability of the maternal condition is addressed. Low-risk patients can be reassured that pregnancy is not contraindicated and that management by the primary physician is appropriate. Women at moderate risk should be evaluated at specialised centres, with management coordinated between the primary physician and specialty centre. Women at high risk of maternal and fetal complications are best cared for at centres with an experienced multidisciplinary team.

\section{Pre-pregnancy intervention}

In high-risk women, who present for evaluation before a planned pregnancy, treatment is recommended, particularly if the valve lesion is amenable to percutaneous intervention or surgical repair, without valve replacement. In addition to decreasing pregnancy-associated risks, intervention before a planned pregnancy may be advantageous because many patients caring for a newborn are reluctant to undergo cardiac surgery after delivery. When valve replacement is the only option, many patients and clinicians opt for a tissue prosthesis, despite limited durability, to avoid the risks of chronic anticoagulation during pregnancy.

\section{Management during pregnancy}

Patients with valvular heart disease respond differently to the haemodynamics of pregnancy depending on the underlying valvular pathology. Increases in heart rate, blood volume and 
Box 2 Cardiac findings in a normal pregnancy

- Normal history

- Fatigue

- Decreased exercise tolerance

- Palpitations

- Lower extremity oedema

- Orthopnoea

- Normal examination

- Midsystolic murmur at left base (pulmonic flow murmur)

- Continuous murmur (mammary souffle)

- Split S1

- Distended neck veins with prominent $a$ and $v$ waves

- Lower extremity oedema

cardiac output are poorly tolerated in women with left-sided obstructive lesions. On the other hand, the decrease in systemic vascular resistance often benefits women with regurgitant lesions until delivery, when the abrupt increase in vascular resistance may precipitate pulmonary oedema. Women with pulmonary hypertension are particularly intolerant of the haemodynamic changes of pregnancy and represent an exceptionally high-risk group.

The type and frequency of cardiac monitoring during pregnancy is determined by the specific valve lesion and clinical course in each patient. It is important to educate patients about symptoms and signs of cardiac decompensation and emphasise the importance of seeking medical attention promptly if these symptoms occur. Even when pregnancy is initially well tolerated, additional cardiac demands, such as infection, anaemia, arrhythmias, pulmonary embolus or simply pain and anxiety, often result in clinical deterioration and require aggressive treatment. In addition to treatment of the exacerbating factor, cardiac demand is minimised by bedrest and oxygen. Judicious medical treatment to decrease heart rate or improve loading conditions, often guided by invasive monitoring, is also appropriate. Preload-dependent lesions may benefit from the lateral decubitus position, both during late pregnancy and during labour and delivery, to prevent reduced venous return owing to compression of the inferior vena cava by the gravid uterus.

\section{Use of drugs}

Avoiding all drugs is not always possible in pregnant women with valvular disease, particularly when heart failure, significant arrhythmias or a prosthetic valve is present. Although no drugs are truly safe in pregnant women, treatment may be essential to maintain cardiac stability. Medications that are contraindicated during pregnancy include ACE inhibitors, angiotensin receptor blockers, amiodarone and nitroprusside, so a transition to alternate treatment before pregnancy is desirable. Cardiac drugs that are commonly used during pregnancy include $\beta$-blockers, hydralazine, diuretics and digoxin. ${ }^{13} 14$ The optimal approach to anticoagulation for a mechanical heart valve during pregnancy remains controversial, as discussed following.

\section{Labour and delivery}

In a woman with valvular disease, a short and pain-free labour and delivery helps to minimise haemodynamic fluctuation. Particularly with severe left-sided valve stenosis, the rapid changes in heart rate, cardiac output, venous return and vascular resistance are difficult to manage, often requiring
Box 3 Basic management principles for pregnant women with valvular disease

\section{Risk assessment}

- Preconceptual

- History of cardiac symptoms including arrhythmias

- Baseline exercise tolerance and functional class (exercise testing, if needed)

- Baseline echocardiogram

- Anatomy and haemodynamics of valve lesion

- Ventricular function and pulmonary pressures

- Stability of cardiac haemodynamics over time

- During pregnancy

- Careful frequent history and physical examination at least once per trimester

- More frequent monitoring if new symptoms develop

- Changes in functional class

- Serial echocardiography for any changes in symptoms or signs

\section{Treatment}

- Preconceptual

- Effective contraception until pregnancy is desired

- Consider valve repair or replacement if symptoms exist before conception

- Adjust drugs as needed to prevent adverse fetal effects

- During pregnancy

- Change to only necessary drugs that are not contraindicated in pregnancy

- Control symptoms with medical treatment, bedrest and oxygen

- Valvuloplasty, if necessary and appropriate

- Valve repair or replacement for uncontrolled class III or IV symptoms

- Labour and delivery

- Short vaginal delivery with excellent anaesthesia

- Left lateral decubitus position

- Caesarean section per obstetric indications

- Invasive monitoring if needed

- Medical treatment to optimise loading conditions and to treat pulmonary oedema

- Post partum

- Treat anaemia

- Medical treatment to optimise loading conditions and to treat pulmonary oedema

- Valve repair or replacement, if indicated

- Counselling and contraception

haemodynamic monitoring, including continuous monitoring of oxygen saturation, ECG, arterial pressure, pulmonary artery and wedge pressures, and cardiac output. Fetal monitoring is another means of assessing the adequacy of cardiac treatment because fetal distress is an indicator of impaired cardiac output.

Women with valvular disease are best managed with a vaginal delivery with adequate pain control. Caesarean section results in greater haemodynamic changes and more blood loss, 
Box 4 Risk stratification for pregnant women with valvular disease

High risk of adverse maternal and fetal outcomes

- Prior cardiac event or arrhythmia

- New York Heart Association class $>2$ or cyanosis

- Systemic ventricular dysfunction (ejection fraction $<40 \%$ )

- Pulmonary hypertension (pulmonary arterial systolic pressure $>50 \%$ systemic pressure)

- Left heart obstruction

- Severe aortic stenosis (valve area $<1 / \mathrm{cm}^{2}$, Doppler jet velocity $>4 \mathrm{~m} / \mathrm{s}$ )

- Symptomatic or severe mitral stenosis

- Severe aortic or mitral regurgitation with NYHA class III or IV symptoms

Low risk of adverse maternal and fetal outcomes

- Asymptomatic mild to moderate stenosis or regurgitation

- Regurgitation with normal left ventricular size and function.

Adapted from Siu et al, ${ }^{12}$ Reimold and Rutherford, ${ }^{5}$ Bonow et al. ${ }^{12}$

and so is typically reserved for obstetric indications. Labour is induced when the cervix is favourable to ensure that all of the appropriate medical staff are available in case any complications develop.

Adequate anaesthesia includes both anxiolytics and narcotics to minimise tachycardia and hypertension due to pain and anxiety. Maternal pushing is minimised and forceps or vacuum-assisted deliveries are often necessary to avoid the sudden rise in systemic vascular resistance and drop in systemic venous return that occurs with maternal pushing. We favour endocarditis prophylaxis for vaginal deliveries ${ }^{15}$ in women with valvular disease because complications such as episiotomies are often not anticipated.

\section{SPECIFIC VALVE LESIONS \\ Mitral stenosis}

The most common cause of mitral stenosis is rheumatic valvular disease, which is often first diagnosed during pregnancy. In pregnant women with mitral stenosis, the increase in cardiac output combined with a decrease in filling time due to increased heart rate can result in increased left atrial pressures and pulmonary oedema. Even in women who were previously asymptomatic, further shortening of the diastolic filling period owing to atrial fibrillation or comorbid conditions that further increase heart rate, such as anaemia or fever, often causes haemodynamic decompensation (fig 2).

The goals of treatment are to (1) treat any underlying condition such as infection, fever or anaemia; (2) slow heart rate to prolong the diastolic filling time; (3) decrease preload, if pulmonary oedema has developed; and (4) maintain blood pressure. Thus, treatment typically includes bedrest, oxygen, $\beta$-blockers, diuretics and antibiotics, if infection is present. Prophylaxis to prevent recurrent rheumatic fever is also needed in most pregnant women with mitral stenosis.

When haemodynamic compromise persists despite appropriate medical treatment, percutaneous balloon valvuloplasty may be needed. The benefit of restoring normal placental blood flow outweighs the procedural risk, and the radiation risk to the fetus is minimised using a lead apron. ${ }^{16}{ }^{17}$ In women with concurrent mitral regurgitation or other contraindications to balloon valvuloplasty, mitral valve surgery may be needed in extreme cases when decompensation persists despite aggressive medical treatment. Although cardiac surgery is avoided in pregnancy whenever possible, there are reports of successful procedures. ${ }^{18}{ }^{19}$ Specific risk to the fetus is difficult to ascertain from the literature, but does not seem to be directly related to gestational age or the duration of cardiopulmonary bypass. ${ }^{18}$ The mother's surgical risk is slightly increased compared with the non-gravid state, largely owing to the emergent nature of the surgery.

\section{Aortic stenosis}

Severe aortic stenosis may be difficult to manage during pregnancy (fig 3). Certainly, all women with symptomatic aortic stenosis should undergo prompt intervention before pregnancy. Most asymptomatic patients tolerate pregnancy well, but a minority develop symptoms of heart failure, angina or syncope. Medical management of symptoms is challenging and includes bedrest, oxygen, treatment of exacerbating factors, $\beta$-blockers and cautious diuresis if volume overload is present. Drugs that decrease afterload may be hazardous, because of the relatively fixed obstruction at the valve level. In patients with persistent haemodynamic compromise, percutaneous valvotomy is an option, depending on the exact morphology of the congenitally abnormal aortic valve. ${ }^{20}$ As with mitral stenosis, surgical valve replacement is considered when the mother's life is in danger.

\section{Pulmonic stenosis}

Pulmonic stenosis may occur in isolation or as a part of other congenital abnormalities such as tetralogy of Fallot. Pulmonary stenosis is generally well tolerated in the absence of other haemodynamically significant lesions. It is also amenable to percutaneous valvuloplasty if necessary.

\section{Aortic or mitral regurgitation}

Patients with chronic left-sided valve regurgitation often do well during pregnancy due to the decrease in afterload, but experience difficulty during labour, delivery and the early postpartum period due to the increase in both venous return and vascular resistance (fig 4). In the peripartum period, diuresis may be needed, and afterload reduction may be helpful in the first $24-48 \mathrm{~h}$ post partum.

In contrast with chronic disease, acute valve regurgitation is not well tolerated. As in the non-pregnant women, acute aortic regurgitation-for example, owing to aortic dissection or aortic valve endocarditis-is a surgical emergency. Women with acute mitral regurgitation, due to a ruptured chord for example, may initially be stabilised with an intra-aortic balloon pump, but typically require urgent surgery.

\section{Pulmonic regurgitation}

Pulmonic regurgitation is most often encountered in patients who have undergone prior intervention for congenital abnormalities such as tetralogy of Fallot. These patients are typically also at a higher risk for arrhythmias. Pulmonary insufficiency is generally well tolerated if the patient is asymptomatic and the right ventricle has normal systolic function and is not significantly dilated. Those patients who have symptoms, and severely enlarged or dysfunctional right ventricles will benefit from valve replacement before pregnancy. Symptoms during pregnancy are generally amenable to diuretic treatment and antiarrhythmic treatment when needed..$^{21} 22$

\section{Prosthetic valves}

There has been concern in the past that bioprosthetic valves may experience accelerated degeneration as a consequence of pregnancy. However, the rate of bioprosthetic valve 

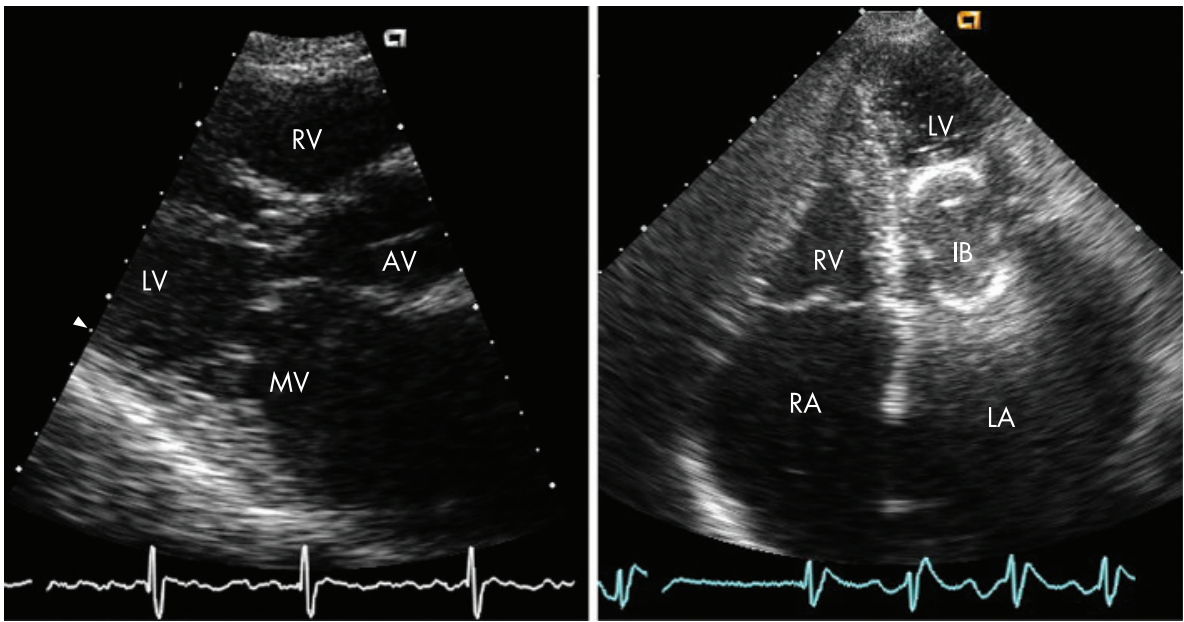

Figure 2 This 23-year-old $G_{1} P_{0}$ woman presented with dyspnoea at 22 weeks gestation. The parasternal long axis view (top) shows rheumatic mitral stenosis with thickening of the leaflet tips and diastolic doming, consistent with commissural fusion. Initial treatment with diuretics and $\beta$-blockers improved her symptoms, but at 28 weeks she decompensated further and underwent balloon valvuloplasty with marked improvement in haemodynamics. The apical four-chamber view (below) shows the Inove valvuloplasty balloon (IB) inflated across the mitral valve. A healthy infant was delivered and the patient remains asymptomatic. Ao, aorta; $\mathrm{AV}$, aortic valve; LA, left atrium; LV, left ventricle; $M V$, mitral valve; RA, right atrium; RV, right ventricle.

degeneration is inversely related to age, and women often have had the valve in place for several years before pregnancy; hence, it is likely that bioprosthetic valve degeneration during pregnancy simply represents the expected longevity of these valves. ${ }^{423}$ Management of women with bioprosthetic valves is similar to management of women with native valvular disease.

The major difficulty in management of women with mechanical prostheses during pregnancy is the anticoagulation requirement. Pregnancy is a thrombogenic state, and so pregnant women with a mechanical prosthesis are at increased risk. The ideal goal is continuous effective anticoagulation that is safe for both the mother and fetus (fig 5). Unfortunately, currently there is no drug that meets this goal. ${ }^{24}$ Warfarin is teratogenic, particularly in the first trimester, with an estimated risk of fetal defects between $5-10 \%,{ }^{13}$ although the risk may be lower when the daily dose is $\leqslant 5 \mathrm{mg} .{ }^{25}$ Warfarin is also associated with an increased risk of fetal intracerebral haemorrhage. Heparin is safer for the fetus because it does not cross the placenta and may be given either subcutaneously or by continuous infusion, but may be less safe for the mother due to difficulty in ensuring adequate anticoagulation. ${ }^{12} 26-28$ Pregnancy induces a hypercoagulable state so that subtherapeutic dosing and thrombosis is a risk with either warfarin or heparin anticoagulation; hence, higher levels of anticoagulation and more frequent monitoring are essential.

There is clearly no preferred approach to anticoagulation in pregnancy. All advocate heparin from week 36 until delivery. Earlier in pregnancy, options include:

(1) heparin for the first trimester, then warfarin until week 36;

(2) heparin throughout pregnancy;

(3) warfarin until week 36

Given the uncertainty surrounding anticoagulation during pregnancy, a detailed discussion is held with the patient regarding the different options. Warfarin is safer for the
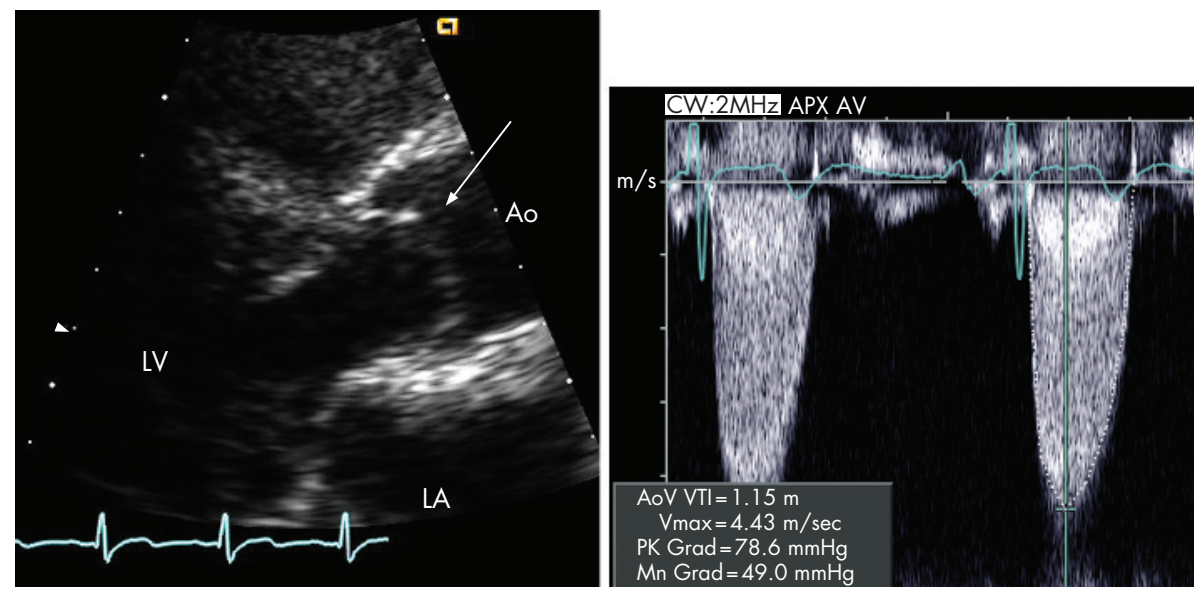

Figure 3 This 21 -year-old $G_{1} P_{0}$ with known severe aortic stenosis developed dyspnoea with exertion at 36 weeks gestation. Echocardiography in a parasternal long-axis view shows a congenitally stenotic aortic valve with doming of the leaflets (arrow) in systole. The continuous-wave Doppler velocity is $4.4 \mathrm{~m} / \mathrm{s}$, with a mean transaortic gradient of $49 \mathrm{~mm} \mathrm{Hg}$ and a valve area of $0.7 \mathrm{~cm}^{2}$. She was placed on bedrest and underwent delivery with guidance of invasive haemodynamic monitoring. Postpartum pulmonary oedema was treated with diuretics, and she underwent aortic valve replacement 2 weeks after delivery. Ao, aorta; AoV VTI, aortic valve velocity time integral; LA, left atrium; LV, left ventricle; Mn Grad, mean gradient; PK Grad, peak gradient; Vmax, maximum velocity. 

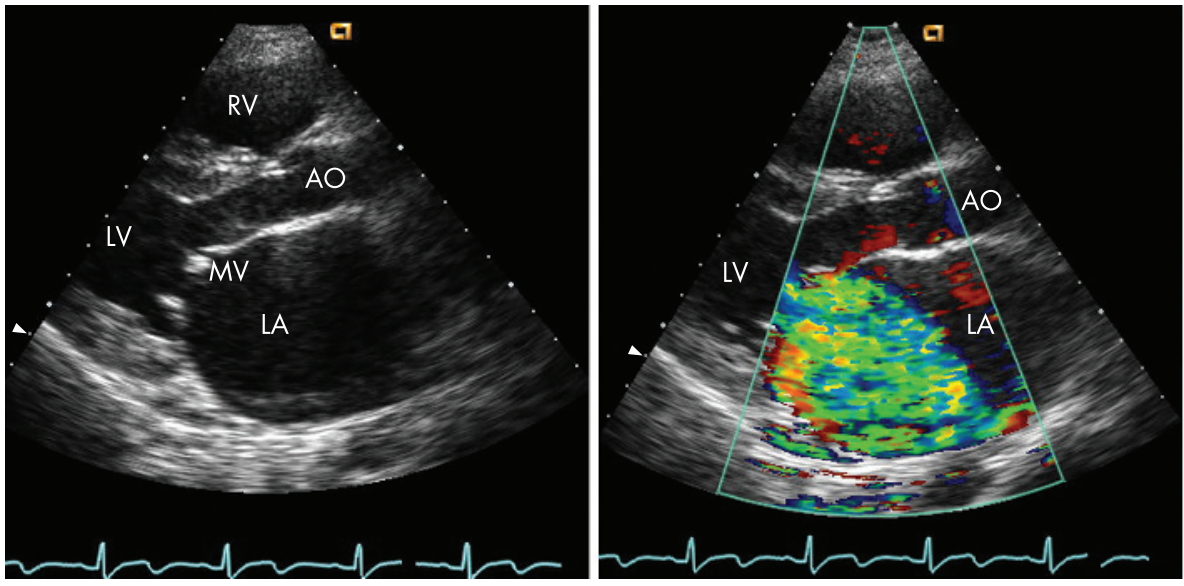

Figure 4 Parasternal views of a 21-year-old woman with rheumatic mitral valvular disease and severe mitral regurgitation. She was medically managed through her pregnancy with diuretics, oxygen and bedrest, and following delivery underwent mitral valve replacement. Two-dimensional views show rheumatic mitral valvular disease and colour Doppler shows severe mitral regurgitation into a severely dilated left atrium. Ao, aorta; $A V$, aortic valve; LA, left atrium; $\mathrm{LV}$, left ventricle; $M V$, mitral valve; RA, right atrium; $\mathrm{RV}$, right ventricle.

mother, but riskier for the fetus, and the ideal method for delivering heparin is yet to be fully elucidated. When warfarin is used, the dose is adjusted to maintain an international normalised ratio in the therapeutic range for the specific valve type as per established guidelines, with more frequent international normalised ratio monitoring than in a non-pregnant patient.

When heparin is used, the options include continuous intravenous unfractionated (UFH) heparin through an indwelling venous line or subcutaneous UFH or low molecular weight heparin. The most important principle to be adhered to while using heparin is that the dose must be adjusted to maintain therapeutic anticoagulation. With UFH, an activated partial thromboplastin time 2.5 times the control is recommended. Continuous intravenous heparin has the disadvantages of risk of infection, cost and inconvenience; subcutaneous heparin requires careful dose adjustment and monitoring.
The use of low molecular weight heparin remains controversial. The prolonged therapeutic effect makes it an attractive alternative to UFH, but early studies suggested an increase in the risk of valve thrombosis in pregnant women with mechanical valves. ${ }^{29-31}$ More recent studies suggest that the increased rate of thrombosis was related to inadequate dosing and lack of monitoring. If used, dosing should be set at twice daily with a $4 \mathrm{~h}$ post-dosing anti-factor Xa level of 1.0-1.2 to ensure adequate therapeutic effect.

\section{Authors' affiliations}

Karen K Stout, Catherine M Otto, Division of Cardiology, Department of Medicine, University of Washington, Seattle, Washington, USA

Competing interests: None.
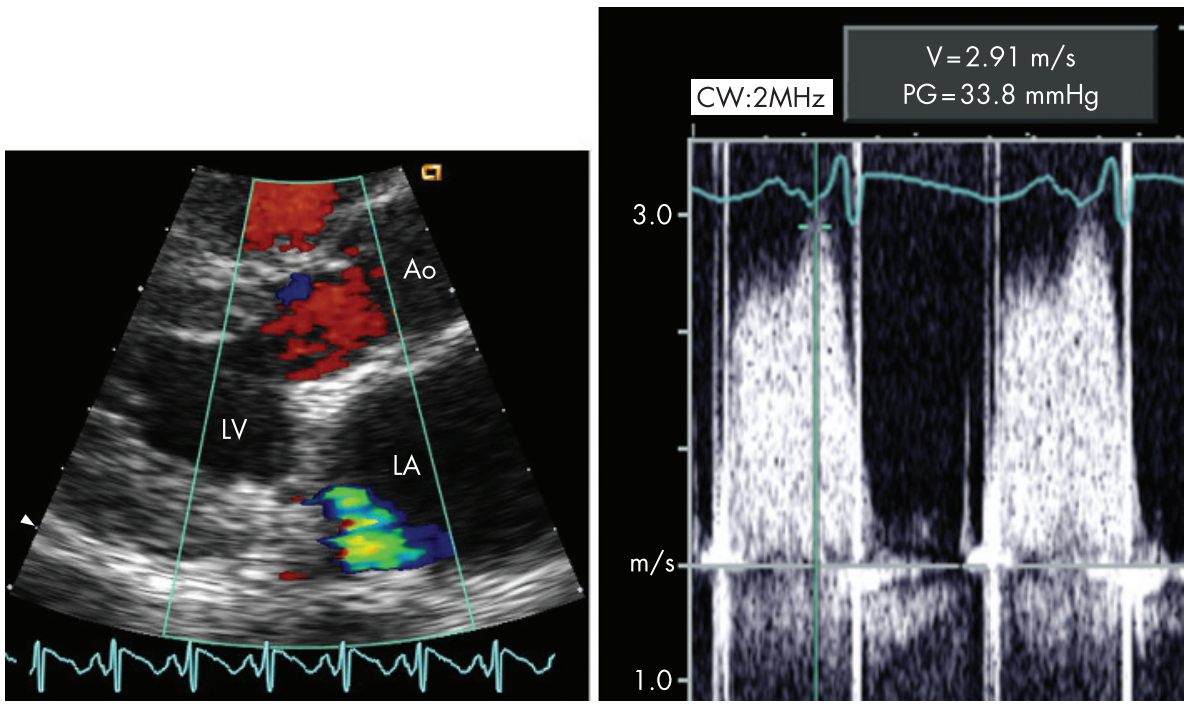

Figure 5 Parasternal long-axis view and Doppler evaluation in a 19-year-old $G_{1} P_{0}$ with a mechanical mitral prosthesis, who developed acute-onset dyspnoea and orthopnoea at 11 weeks gestation while on fixed-dose subcutaneous heparin. The parasternal long-axis image shows an immobile mechanical prosthesis with mild regurgitation. The Doppler signal, recorded from an apical approach, shows severe stenosis with a mean transmitral diastolic gradient of $25 \mathrm{~mm} \mathrm{Hg}$. At 3 weeks gestation, her treatment had been changed from coumadin to subcutaneous heparin. Evaluation showed acute mitral valve thrombosis. The patient elected to proceed with emergent valve replacement rather than thrombolytics, owing to the risk of a cerebrovascular event. The fetus was miscarried and the patient recovered uneventfully from her repeat mitral valve replacement. Ao, aorta; $A V$, aortic valve; LA, left atrium; $\mathrm{LV}$, left ventricle; $M V$, mitral valve; $\mathrm{PG}$, pressure gradient; RA, right atrium; RV, right ventricle; $\mathrm{V}$, velocity. 


\section{REFERENCES}

1 Siu SC, Sermer M, Colman JM, et al. Prospective multicenter study of pregnancy outcomes in women with heart disease. Circulation 2001;104:515-21.

2 Siu SC, Colman JM, Sorensen S, et al. Adverse neonatal and cardiac outcomes are more common in pregnant women with cardiac disease. Circulation 2002; 105:2179-84.

3 Soler-Soler J, Galve E. Worldwide perspective of valve disease. Heart 2000;83:721-5.

4 Dore A, Somerville J. Pregnancy in patients with pulmonary autograft valve replacement. Eur Heart J 1997;18:1659-62.

5 Reimold SC, Rutherford JD. Clinical practice. Valvular heart disease in pregnancy. N Engl J Med 2003;349:52-9.

6 Thorne SA. Pregnancy in heart disease. Heart 2004;90:450-6.

7 van Oppen AC, Stigter RH, Bruinse HW. Cardiac output in normal pregnancy: a critical review. Obstet Gynecol 1996;87:310-18.

8 Easterling TR, Carlson KL, Schmucker BC, et al. Measurement of cardiac output in pregnancy by Doppler technique. Am J Perinatol 1990;7:220-2.

9 Robson SC, Hunter S, Moore M, et al. Haemodynamic changes during the puerperium: a Doppler and M-mode echocardiographic study. $\mathrm{Br} J \mathrm{Obstet}$ Gynaecol 1987;94:1028-39.

10 Easterling TR, Benedetti TJ, Schmucker BC, et al. Maternal hemodynamics in normal and preeclamptic pregnancies: a longitudinal study [see comments] Obstet Gynecol 1990;76:1061-9.

11 Robson SC, Dunlop W, Boys RJ, et al. Cardiac output during labour. BMJ (Clin Res Ed) 1987;295:1169-72.

12 Bonow RO, Carabello BA, deleon AC, et al. ACC/AHA guidelines for the management of patients with valvular heart disease: a report of the American College of Cardiology/American Heart Association Task Force on Practice Guidelines (Committee on management of patients with valvular heart disease) J Am Coll Cardiol 1998;32:1486-588.

13 Qasqas SA, McPherson C, Frishman WH, et al. Cardiovascular pharmacotherapeutic considerations during pregnancy and lactation. Cardiol Rev 2004; 12:201-21

14 Qasqas SA, McPherson C, Frishman WH, et al. Cardiovascular pharmacotherapeutic considerations during pregnancy and lactation. Cardiol $\operatorname{Rev} 2004 ; 12: 240-61$

15 Dajani AS, Taubert KA, Wilson W, et al. Prevention of bacterial endocarditis. Recommendations by the American Heart Association. JAMA 1997; 277:1794-801.

16 Patel JJ, Mitha AS, Hassen F, et al. Percutaneous balloon mitral valvotomy in pregnant patients with tight pliable mitral stenosis. Am Heart J 1993;125:1106-9.
17 Ribeiro PA, Fawzy ME, Awad M, et al. Balloon valvotomy for pregnant patients with severe pliable mitral stenosis using the Inove technique with total abdominal and pelvic shielding. Am Heart J 1992;124:1558-62.

18 Weiss BM, von Segesser LK, Alon E, et al. Outcome of cardiovascular surgery and pregnancy: a systematic review of the period 1984-1996. Am J Obstet Gynecol 1998; 179:1643-53.

19 Sullivan HJ. Valvular heart surgery during pregnancy. Surg Clin North Am 1995;75:59-75.

20 Myerson SG, Mitchell AR, Ormerod OJ, et al. What is the role of balloon dilatation for severe aortic stenosis during pregnancy? J Heart Valve Dis 2005; 14:147-50

21 Veldtman GR, Connolly HM, Grogan M, et al. Outcomes of pregnancy in women with tetralogy of Fallot. J Am Coll Cardiol 2004:44:174-80.

22 Meijer JM, Pieper PG, Drenthen W, et al. Pregnancy, fertility, and recurrence risk in corrected tetralogy of Fallot. Heart 2005;91:801-5.

23 Briand M, Dumesnil JG, Kadem L, et al. Reduced systemic arterial compliance impacts significantly on left ventricular afterload and function in aortic stenosis: implications for diagnosis and treatment. J Am Coll Cardiol 2005;46:291-8.

24 Hung L, Rahimtoola SH. Prosthetic heart valves and pregnancy. Circulation 2003;107:1240-6.

25 Vitale N, De Feo M, De Santo LS, et al. Dose-dependent fetal complications of warfarin in pregnant women with mechanical heart valves. J Am Coll Cardiol 1999;33:1637-41.

26 Salem DN, Stein PD, Al Ahmad A, et al. Antithrombotic therapy in valvular heart disease-native and prosthetic: the Seventh ACCP Conference on antithrombotic and thrombolytic therapy. Chest 2004; 126:457S-82S.

27 Elkayam $\mathrm{U}$, Singh $\mathrm{H}$, Irani $\mathrm{A}$, et al. Anticoagulation in pregnant women with prosthetic heart valves. J Cardiovasc Pharmacol Ther 2004:9:107-15.

28 Gohlke-Barwolf C, Acar J, Oakley C, et al. Guidelines for prevention of thromboembolic events in valvular heart disease. Study Group of the Working Group on Valvular Heart Disease of the European Society of Cardiology. Eur Heart J 1995; 16:1320-30.

29 Chan WS, Anand S, Ginsberg JS. Anticoagulation of pregnant women with mechanical heart valves: a systematic review of the literature. Arch Intern Med 2000;160:191-6

30 Ginsberg JS, Chan WS, Bates SM, et al. Anticoagulation of pregnant women with mechanical heart valves. Arch Intern Med 2003;163:694-8.

31 Elkayam U, Bitar F. Valvular heart disease and pregnancy part I: native valves. J Am Coll Cardiol 2005;46:223-30.

\section{bmjupdates+}

bmjupdates+ is a unique and free alerting service, designed to keep you up to date with the medical literature that is truly important to your practice.

bmjupdates+ will alert you to important new research and will provide you with the best new evidence concerning important advances in health care, tailored to your medical interests and time demands.

Where does the information come from?

bmjupdates+ applies an expert critical appraisal filter to over 100 top medical journals A panel of over 2000 physicians find the few 'must read' studies for each area of clinical interest

Sign up to receive your tailored email alerts, searching access and more...

www.bmjupdates.com 